\title{
On the minimum of independent geometrically distributed random variables
}

\author{
Gianfranco Ciardo \\ Lawrence Leemis \\ William \& Mary, Imleem@wm.edu \\ David Nicol
}

Follow this and additional works at: https://scholarworks.wm.edu/aspubs

Part of the Mathematics Commons

\section{Recommended Citation}

Ciardo, Gianfranco; Leemis, Lawrence; and Nicol, David, On the minimum of independent geometrically distributed random variables (1995). Statistics and Probability Letters, 23(4), 313-326.

https://doi.org/10.1016/0167-7152(94)00130-Z

This Article is brought to you for free and open access by the Arts and Sciences at W\&M ScholarWorks. It has been accepted for inclusion in Arts \& Sciences Articles by an authorized administrator of W\&M ScholarWorks. For more information, please contact scholarworks@wm.edu. 


\title{
On the minimum of independent geometrically distributed random variables *
}

\author{
Gianfranco Ciardo $^{1} \quad$ Lawrence M. Leemis ${ }^{2} \quad$ David Nicol $^{1}$ \\ ${ }^{1}$ Department of Computer Science $\quad{ }^{2}$ Department of Mathematics \\ College of William and Mary \\ Williamsburg, VA 23187-8795, USA \\ $\{$ ciardo, leemis, nicol\}@cs.wm.edu
}

Abstract: The expectations $E\left[X_{(1)}\right], E\left[Z_{(1)}\right]$, and $E\left[Y_{(1)}\right]$ of the minimum of $n$ independent geometric, modified geometric, or exponential random variables with matching expectations differ. We show how this is accounted for by stochastic variability and how $E\left[X_{(1)}\right] / E\left[Y_{(1)}\right]$ equals the expected number of ties at the minimum for the geometric random variables. We then introduce the "shifted geometric distribution", and show that there is a unique value of the shift for which the individual shifted geometric and exponential random variables match expectations both individually and in their minimums.

Keywords: Geometric distribution; exponential distribution; stochastic ordering; order statistics.

*Address for correspondence: Gianfranco Ciardo, Department of Computer Science, College of William and Mary, P.O. Box 8795, Williamsburg, VA 23187-8795, USA. This research was partially supported by the National Aeronautics and Space Administration under NASA Contract No. NAS1-19480 while the authors were in residence at the Institute for Computer Applications in Science and Engineering (ICASE), NASA Langley Research Center, Hampton, VA 23681. 


\section{Introduction}

The purpose of this note is to compare the distributions of the minimums of two sets of random variables, respectively with geometric and exponential distributions, having pairwise matching means. The geometric distribution is the discrete analog of the exponential distribution and can be applied to a variety of performance models which can be analyzed by analytic or simulation methods. The following notation is used:

- $\mathbb{N}=\{0,1,2, \ldots\}$, the natural numbers.

- $\mathbb{N}^{+}=\{1,2,3, \ldots\}$, the positive natural numbers.

- $N=\{1,2, \ldots, n\}$, the first $n$ natural numbers ( $n$ is a constant which will be clear from the context).

- $F_{A}(t)=\operatorname{Pr}\{A \leq a\}$, the cumulative distribution function (CDF) of a random variable A.

- $\bar{F}_{A}(t)=1-F_{A}(t)$, the complement of the CDF of $A$ (its survivor function).

\section{Geometric, modified geometric, and exponential dis- tributions}

Two random variables $X$ and $Z$ are said to have a geometric distribution with parameter $\alpha \in(0,1), X \sim \operatorname{Geom}(\alpha)$, and a modified geometric distribution with parameter $\beta \in(0,1)$, $Z \sim \operatorname{ModGeom}(\beta)$, if their probability mass functions (pmfs) are, respectively (Trivedi, 1982),

$$
\begin{gathered}
\forall k \in \mathbb{N}^{+}, \operatorname{Pr}\{X=k\}=\alpha(1-\alpha)^{k-1} \text { and } \\
\forall k \in \mathbb{N}, \operatorname{Pr}\{Z=k\}=\beta(1-\beta)^{k},
\end{gathered}
$$

from which it follows that their CDFs at the mass values are

$$
\forall k \in \mathbb{N}^{+}, \operatorname{Pr}\{X \leq k\}=\sum_{l=1}^{k} \alpha(1-\alpha)^{l-1}=1-(1-\alpha)^{k} \text { and }
$$




$$
\forall k \in \mathbb{N}, \operatorname{Pr}\{Z \leq k\}=\sum_{l=0}^{k} \beta(1-\beta)^{l}=1-(1-\beta)^{k+1},
$$

and that their expectations are

$$
\begin{gathered}
E[X]=\sum_{k=1}^{\infty} k \alpha(1-\alpha)^{k-1}=\frac{1}{\alpha} \text { and } \\
E[Z]=\sum_{k=0}^{\infty} k \beta(1-\beta)^{k}=\frac{1-\beta}{\beta} .
\end{gathered}
$$

Informally, the difference between a geometric and a modified geometric distribution with the same parameter is the way in which they count: the geometric distribution starts at one, the modified geometric distribution starts at zero. Hence, if $X \sim \operatorname{Geom}(\alpha), X-$ $1 \sim \operatorname{Mod} \operatorname{Geom}(\alpha)$. Equivalently, the geometric distribution models the trial number of the first "success" in repeated independent Bernoulli trials, whereas the modified geometric distribution models the number of trials before the first success.

The above assumes that the "time-step" of the distribution is the same as the units in which time is measured. This restriction is removed by considering $X$ and $Z$ as random variables assuming values in $\left\{k \omega: k \in \mathbb{N}^{+}\right\}$or $\{k \omega: k \in \mathbb{N}\}$, respectively, for some time-step $\omega>0$ :

$$
X \sim \operatorname{Geom}(\alpha, \omega) \Longleftrightarrow \forall t \in \mathbb{R}, \operatorname{Pr}\{X \leq t\}= \begin{cases}1-(1-\alpha)^{\left\lfloor\frac{t}{\omega}\right\rfloor} & \text { if } t \geq 0 \\ 0 & \text { otherwise }\end{cases}
$$

and

$$
Z \sim \operatorname{Mod} \operatorname{Geom}(\beta, \omega) \Longleftrightarrow \forall t \in \mathbb{R}, \operatorname{Pr}\{Z \leq t\}= \begin{cases}1-(1-\beta)^{\left\lfloor\frac{t}{\omega}\right\rfloor+1} & \text { if } t \geq 0 \\ 0 & \text { otherwise }\end{cases}
$$

which imply

$$
\begin{gathered}
\forall k \in \mathbb{N}^{+}, \operatorname{Pr}\{X=k \omega\}=\alpha(1-\alpha)^{k-1} \text { and } \\
\forall k \in \mathbb{N}, \operatorname{Pr}\{Z=k \omega\}=\beta(1-\beta)^{k}
\end{gathered}
$$

and

$$
\begin{gathered}
E[X]=\frac{\omega}{\alpha} \text { and } \\
E[Z]=\frac{\omega(1-\beta)}{\beta} .
\end{gathered}
$$


It is well known that both the geometric and modified geometric distributions are discrete analogs of the exponential distribution. In particular, given an exponential random variable $Y$ with rate $\lambda>0$,

$$
Y \sim \operatorname{Expo}(\lambda) \Longleftrightarrow \forall t \geq 0, \operatorname{Pr}\{Y \leq t\}=1-e^{-\lambda t} \Rightarrow E[Y]=\lambda^{-1}
$$

one can determine $\alpha$ and $\beta$ so that $X$ and $Z$ match $Y$ in expectation:

$$
\begin{gathered}
E[X]=\frac{\omega}{\alpha}=\lambda^{-1}=E[Y] \Rightarrow \alpha=\lambda \omega \text { and } \\
E[Z]=\frac{\omega(1-\beta)}{\beta}=\lambda^{-1}=E[Y] \Rightarrow \beta=\frac{\lambda \omega}{1+\lambda \omega},
\end{gathered}
$$

and then, using these values for $\alpha$ and $\beta$, the distributions of $X$ and $Z$ approximate that of $Y$ arbitrarily well as the time-step $\omega$ is reduced:

$$
\begin{gathered}
\lim _{\omega \downarrow 0} \operatorname{Pr}\{X \leq t\}=\lim _{\omega \downarrow 0} 1-(1-\lambda \omega)^{\left\lfloor\frac{t}{\omega}\right\rfloor}=1-e^{-\lambda t}=\operatorname{Pr}\{Y \leq t\} \text { and } \\
\lim _{\omega \downarrow 0} \operatorname{Pr}\{Z \leq t\}=\lim _{\omega \downarrow 0} 1-\left(1-\frac{\lambda \omega}{1+\lambda \omega}\right)^{\left\lfloor\frac{t}{\omega}\right\rfloor+1}=1-e^{-\lambda t}=\operatorname{Pr}\{Y \leq t\} .
\end{gathered}
$$

Note that $\alpha=\lambda \omega \in(0,1)$ implies $\omega<\lambda^{-1}$, that is, it is not possible to match the mean of an exponential random variable $Y \sim \operatorname{Expo}(\lambda)$ with a geometric random variable having a time-step $\omega>\lambda^{-1}$. In the special case $\omega=\lambda^{-1}, \alpha=1$ and the distribution of $X$ degenerates to a constant: $X \sim \operatorname{Geom}(1, \omega) \equiv \operatorname{Const}(\omega)$. In the following, we allow this case and require

$\omega \in\left(0, \lambda^{-1}\right]$. No such restriction exists in the case of the modified geometric distribution, where any $\omega>0$ can be used.

\section{The minimum of a set of random variables}

Consider now three sets of $n \geq 2$ independent random variables, $\left\{X_{i}: i \in N\right\},\left\{Z_{i}: i \in N\right\}$, and $\left\{Y_{i}: i \in N\right\}$ with matching means. Given $\lambda_{1}, \ldots, \lambda_{n}, \omega>0$,

$$
\forall i \in N, X_{i} \sim \operatorname{Geom}\left(\alpha_{i}, \omega\right), E\left[X_{i}\right]=\lambda_{i}^{-1} \Rightarrow \alpha_{i}=\lambda_{i} \omega
$$

$\forall i \in N, Z_{i} \sim \operatorname{Mod} \operatorname{Geom}\left(\beta_{i}, \omega\right), E\left[Z_{i}\right]=\lambda_{i}^{-1} \Rightarrow \beta_{i}=\frac{\lambda_{i} \omega}{1+\lambda_{i} \omega}$, and

$$
\forall i \in N, Y_{i} \sim \operatorname{Expo}\left(\lambda_{i}\right), E\left[Y_{i}\right]=\lambda_{i}^{-1}
$$


Since $\omega \in \bigcap_{i \in N}\left(0, \lambda_{i}^{-1}\right]$, we obtain $\omega \in\left(0, \lambda_{M A X}^{-1}\right]$, where $\lambda_{M A X}=\max \left\{\lambda_{i}: i \in N\right\}$.

It is well known that the minimum of each of these sets of random variables has the same type of distribution as the elements of the set (Uppuluri, 1964, Margolin 1967):

$$
\begin{aligned}
& X_{(1)}=\min \left\{X_{i}: i \in N\right\} \quad \sim \operatorname{Geom}\left(1-\prod_{i \in N}\left(1-\alpha_{i}\right), \omega\right) \\
& Z_{(1)}=\min \left\{Z_{i}: i \in N\right\} \quad \sim \operatorname{ModGeom}\left(1-\prod_{i \in N}\left(1-\beta_{i}\right), \omega\right), \text { and } \\
& Y_{(1)}=\min \left\{Y_{i}: i \in N\right\}
\end{aligned}
$$

Hence, $X_{(1)}, Z_{(1)}$, and $Y_{(1)}$ have different expectations:

$$
\begin{aligned}
& E\left[X_{(1)}\right]=\frac{\omega}{1-\prod_{i \in N}\left(1-\alpha_{i}\right)}=\frac{\omega}{1-\prod_{i \in N}\left(1-\lambda_{i} \omega\right)} \neq\left(\sum_{i \in N} \lambda_{i}\right)^{-1}=E\left[Y_{(1)}\right] \text { and } \\
& E\left[Z_{(1)}\right]=\frac{\omega \prod_{i \in N}\left(1-\beta_{i}\right)}{1-\prod_{i \in N}\left(1-\beta_{i}\right)}=\frac{\omega}{-1+\prod_{i \in N}\left(1+\lambda_{i} \omega\right)} \neq\left(\sum_{i \in N} \lambda_{i}\right)^{-1}=E\left[Y_{(1)}\right] .
\end{aligned}
$$

Theorem 1. For $n \geq 2, E\left[X_{(1)}\right]>E\left[Y_{(1)}\right]>E\left[Z_{(1)}\right]$.

Proof. We prove that $E\left[X_{(1)}\right]>E\left[Y_{(1)}\right]$ by induction on $n$, hence we make the index $n$ explicit by writing $E\left[X_{(1, n)}\right]$ and $E\left[Y_{(1, n)}\right]$.

Base step: For $n=2$,

$$
E\left[X_{(1,2)}\right]=\frac{\omega}{1-\left(1-\lambda_{1} \omega\right)\left(1-\lambda_{2} \omega\right)}=\frac{1}{\lambda_{1}+\lambda_{2}-\lambda_{1} \lambda_{2} \omega}>\frac{1}{\lambda_{1}+\lambda_{2}}=E\left[Y_{(1,2)}\right] .
$$

Inductive Hypothesis: Assume that, for a given $n, E\left[X_{(1, n)}\right]>E\left[Y_{(1, n)}\right]$. Then,

$$
\frac{\omega}{1-\prod_{i \in N}\left(1-\lambda_{i} \omega\right)}>\left(\sum_{i \in N} \lambda_{i}\right)^{-1} \Rightarrow \prod_{i \in N}\left(1-\lambda_{i} \omega\right)>1-\sum_{i \in N} \lambda_{i} \omega
$$

Inductive Step: Then $E\left[X_{(1, n+1)}\right]>E\left[Y_{(1 n+1)}\right]$, since

$$
\begin{aligned}
E\left[X_{(1, n+1)}\right] & =\frac{\omega}{1-\left(\prod_{i \in N}\left(1-\lambda_{i} \omega\right)\right)\left(1-\lambda_{n+1} \omega\right)} \\
& >\frac{\omega}{1-\left(1-\sum_{i \in N} \lambda_{i} \omega\right)\left(1-\lambda_{n+1} \omega\right)}
\end{aligned}
$$




$$
=\frac{1}{\sum_{i \in N} \lambda_{i}+\lambda_{n+1}-\left(\sum_{i \in N} \lambda_{i}\right) \lambda_{n+1} \omega}>\left(\sum_{i \in N \cup\{n+1\}} \lambda_{i}\right)^{-1}=E\left[Y_{(1, n+1)}\right]
$$

The proof that $E\left[Z_{(1)}\right]<E\left[Y_{(1)}\right]$ is analogous and is omitted. QED.

In other words, the minimum of $n$ independent exponential random variables is always strictly bounded in expectation by the minimums of $n$ independent geometric and modified geometric random variables with matching means. For example, if $n=2$, and $\lambda_{1}=\lambda_{2}=\lambda$,

$$
E\left[X_{(1)}\right]=(2 \lambda(1-\lambda \omega / 2))^{-1}>E\left[Y_{(1)}\right]=(2 \lambda)^{-1}>E\left[Z_{(1)}\right]=(2 \lambda(1+\lambda \omega / 2))^{-1} .
$$

$E\left[X_{(1)}\right]$ and $E\left[Z_{(1)}\right]$ coincide with $E\left[Y_{(1)}\right]$ only in the limit, as $\omega \downarrow 0$ (see figure 1):

$$
\begin{aligned}
& \lim _{\omega \downarrow 0} E\left[X_{(1)}\right]=\lim _{\omega \downarrow 0} \frac{\omega}{1-\prod_{i \in N}\left(1-\lambda_{i} \omega\right)}=\lim _{\omega \downarrow 0} \frac{\omega}{\sum_{i \in N} \lambda_{i} \omega+o(\omega)}=\left(\sum_{i \in N} \lambda_{i}\right)^{-1}=E\left[Y_{(1)}\right] \text { and } \\
& \lim _{\omega \downarrow 0} E\left[Z_{(1)}\right]=\lim _{\omega \downarrow 0} \frac{\omega}{-1+\prod_{i \in N}\left(1+\lambda_{i} \omega\right)}=\lim _{\omega \downarrow 0} \frac{\omega}{\sum_{i \in N} \lambda_{i} \omega+o(\omega)}=\left(\sum_{i \in N} \lambda_{i}\right)^{-1}=E\left[Y_{(1)}\right] .
\end{aligned}
$$

The convergence of $E\left[X_{(1)}\right]$ and $E\left[Z_{(1)}\right]$ to $E\left[Y_{(1)}\right]$ as $\omega \downarrow 0$ can also be derived observing that

$$
E\left[X_{(1)}\right]-\omega<E\left[Z_{(1)}\right]<E\left[Y_{(1)}\right]<E\left[X_{(1)}\right]<E\left[Z_{(1)}\right]+\omega,
$$

which follows from the fact that $\left(X_{i}-\omega\right) \sim \operatorname{Mod} \operatorname{Geom}\left(\alpha_{i}, \omega\right)$ and $\left(Z_{i}+\omega\right) \sim \operatorname{Geom}\left(\beta_{i}, \omega\right)$, and from $\forall i \in N, \alpha_{i}<\beta_{i}$, which imply that $E\left[X_{(1)}-\omega\right]<E\left[Z_{(1)}\right]$ and $E\left[Z_{(1)}+\omega\right]>E\left[X_{(1)}\right]$.

The next section contains an explanation for these inequalities.

\section{Stochastic variability}

Random variables with the same mean can be compared using the notion of stochastic variability, described in Ross (1983), for which there are two equivalent definitions. $Y$ is said to be stochastically more variable than $X, X \leq_{\mathrm{v}} Y$, if

$$
\forall \text { increasing convex function } g, E[g(X)] \leq E[g(Y)]
$$

or, equivalently, provided $X$ and $Y$ have nonnegative support, if

$$
\forall a \geq 0, \int_{a}^{\infty} \bar{F}_{X}(t) d t \leq \int_{a}^{\infty} \bar{F}_{Y}(t) d t .
$$


An additional useful notion codifies the idea that the remaining lifetime of a nonnegative random variable conditioned on exceeding some value $a$ has never greater expectation (NBUE: New Better Than Used in Expectation), or never smaller expectation (NWUE: New Worse Than Used in Expectation), than the original lifetime. Formally, a nonnegative random variable $A$ is NBUE if

$$
\forall a \geq 0, E[A-a \mid A>a] \leq E[A]
$$

and is NWUE if

$$
\forall a \geq 0, E[A-a \mid A>a] \geq E[A]
$$

Ross lists some important consequences of these definitions:

- If $X$ and $Y$ are nonnegative, $X \leq_{\mathrm{v}} Y$, and $E[X]=E[Y]$, then $-X \leq_{\mathrm{v}}-Y$.

- If $g: \mathbb{R}^{n} \rightarrow \mathbb{R}$ is an increasing convex function, if $\forall i \in N, X_{i} \leq_{\mathbf{v}} Y_{i},\left\{X_{i}: i \in N\right\}$ are independent, and $\left\{Y_{i}: i \in N\right\}$ are independent, then

$$
g\left(X_{1}, X_{2}, \ldots, X_{n}\right) \leq_{\mathrm{v}} g\left(Y_{1}, Y_{2}, \ldots, Y_{n}\right)
$$

- If $X$ is NBUE, and $Y$ is exponential with the same mean as $X$, then $X \leq_{\mathrm{v}} Y$.

- If $Z$ if NWUE and $Y$ is exponential with the same mean as $Z$ then $Y \leq_{\mathrm{v}} Z$.

These last two facts are used to relate $X \sim \operatorname{Geom}(\alpha, \omega), Z \sim \operatorname{ModGeom}(\beta, \omega)$, and $Y \sim$ $\operatorname{Expo}(\lambda)$ with the same mean, by showing that the geometric distribution is NBUE and that the modified geometric distribution is NWUE. Let $X \sim \operatorname{Geom}(\alpha, \omega), Z \sim \operatorname{Mod} \operatorname{Geom}(\beta, \omega)$, and choose any $a \geq 0$. Using the memoryless property of the geometric distribution, we can derive:

$$
\begin{aligned}
E[X-a \mid X>a] & =E[X \mid X>a]-a=\left\lfloor\frac{a}{\omega}\right\rfloor \omega+E[X]-a \leq E[X] \text { and } \\
E[Z-a \mid Z>a] & =E[Z \mid Z>a]-a=\left(\left\lfloor\frac{a}{\omega}\right\rfloor+1\right) \omega+E[Z]-a>E[Z] .
\end{aligned}
$$

Therefore, $X \leq_{\mathrm{v}} Y \leq_{\mathrm{v}} Z$.

Considering again the three sets of independent random variables with matching means $\left\{X_{i}: i \in N\right\},\left\{Z_{i}: i \in N\right\}$, and $\left\{Y_{i}: i \in N\right\}$ observe that

$$
\min \left\{a_{i}: i \in N\right\}=-\max \left\{-a_{i}: i \in N\right\}
$$


Since max is an increasing convex function and $\forall i \in N,-X_{i} \leq_{\mathrm{v}}-Y_{i} \leq_{\mathrm{v}}-Z_{i}$,

$$
\max \left\{-X_{i}: i \in N\right\} \leq_{\mathrm{v}} \max \left\{-Y_{i}: i \in N\right\} \leq_{\mathrm{v}} \max \left\{-Z_{i}: i \in N\right\},
$$

implying that

$$
-E\left[\max \left\{-X_{i}: i \in N\right\}\right] \geq-E\left[\max \left\{-Y_{i}: i \in N\right\}\right] \geq-E\left[\max \left\{-Z_{i}: i \in N\right\}\right],
$$

and thus that

$E\left[\min \left\{X_{i}: i \in N\right\}\right]=E\left[X_{(1)}\right] \geq E\left[\min \left\{Y_{i}: i \in N\right\}\right]=E\left[Y_{(1)}\right] \geq E\left[\min \left\{Z_{i}: i \in N\right\}\right]=E\left[Z_{(1)}\right]$.

\section{Matching the minimums by changing the time-step}

This section presents an explanation for the existence of the strict Inequality (1) in Section 3 , and its quantification, based on the possibility of a tie for the minimum in the set $\left\{X_{i}\right.$ : $i \in N\}$. A confirmation of this intuition is found by defining a new random variable, $W_{(1)}$, obtained dividing $X_{(1)}$ by the expected number of random variables tied for the minimum: the expectation of this "weighted minimum" $W_{(1)}$ is indeed the same as that of $Y_{(1)}$.

The discrete nature of the geometric distribution implies that several random variables in $\left\{X_{i}: i \in N\right\}$ might coincide with $X_{(1)}$. Define $I_{[1]}$ to be the the set of indices among $N$ corresponding to such random variables ( $I_{[1]}$ is itself random):

$$
I_{[1]}=\left\{i \in N: X_{i}=X_{(1)}\right\} \neq \emptyset .
$$

The pmf of $I_{[1]}$ is

$$
\begin{aligned}
\forall s \subseteq N, s \neq \emptyset, \operatorname{Pr}\left\{I_{[1]}=s\right\} & =\operatorname{Pr}\left\{\forall i \in s, X_{i}=X_{(1)} \wedge \forall j \in N \backslash s, X_{j}>X_{(1)}\right\} \\
& =\sum_{k=1}^{\infty} \operatorname{Pr}\left\{\forall i \in s, X_{i}=k \omega \wedge \forall j \in N \backslash s, X_{j}>k \omega\right\} \\
& =\sum_{k=1}^{\infty}\left(\prod_{i \in s} \alpha_{i}\left(1-\alpha_{i}\right)^{k-1}\right)\left(\prod_{j \in N \backslash s}\left(1-\alpha_{j}\right)^{k}\right) \\
& =\frac{\left(\prod_{i \in s} \alpha_{i}\right)\left(\prod_{j \in N \backslash s}\left(1-\alpha_{j}\right)\right)}{1-\prod_{l \in N}\left(1-\alpha_{l}\right)} .
\end{aligned}
$$


This result is more easily obtained observing that, because of the absence of memory of the geometric distribution, $I_{[1]}$ and $X_{(1)}$ are independent, hence $\operatorname{Pr}\left\{I_{[1]}=s\right\}$ is simply the product of the one-step probability of success for the elements of $s$ and of the one-step probability of failure for the elements not in $s$, normalized by the probability that at least one success occurs.

For example, if $n=2$, the three possible values for $I_{[1]}$ and their probabilities are:

$$
\begin{gathered}
\operatorname{Pr}\left\{I_{[1]}=\{1\}\right\}=\operatorname{Pr}\left\{X_{1}<X_{2}\right\}=\frac{\alpha_{1}\left(1-\alpha_{2}\right)}{1-\left(1-\alpha_{1}\right)\left(1-\alpha_{2}\right)}=\frac{\lambda_{1}-\lambda_{1} \lambda_{2} \omega}{\lambda_{1}+\lambda_{2}-\lambda_{1} \lambda_{2} \omega} \\
\operatorname{Pr}\left\{I_{[1]}=\{2\}\right\}=\operatorname{Pr}\left\{X_{1}>X_{2}\right\}=\frac{\alpha_{2}\left(1-\alpha_{1}\right)}{1-\left(1-\alpha_{1}\right)\left(1-\alpha_{2}\right)}=\frac{\lambda_{2}-\lambda_{1} \lambda_{2} \omega}{\lambda_{1}+\lambda_{2}-\lambda_{1} \lambda_{2} \omega} \\
\operatorname{Pr}\left\{I_{[1]}=\{1,2\}\right\}=\operatorname{Pr}\left\{X_{1}=X_{2}\right\}=\frac{\alpha_{1} \alpha_{2}}{1-\left(1-\alpha_{1}\right)\left(1-\alpha_{2}\right)}=\frac{\lambda_{1} \lambda_{2} \omega}{\lambda_{1}+\lambda_{2}-\lambda_{1} \lambda_{2} \omega}
\end{gathered}
$$

In general, the probability that a particular $X_{i}$ is equal $X_{(1)}$, or that $i \in I_{[1]}$, is

$$
\begin{aligned}
\operatorname{Pr}\left\{X_{i}=X_{(1)}\right\} & =\sum_{k=1}^{\infty} \operatorname{Pr}\left\{X_{i}=k \omega \wedge \forall j \in N, j \neq i, X_{j} \geq k \omega\right\} \\
& =\sum_{k=1}^{\infty} \alpha_{i}\left(1-\alpha_{i}\right)^{k-1} \prod_{j \in N, j \neq i}\left(1-\alpha_{j}\right)^{k-1} \\
& =\frac{\alpha_{i}}{1-\prod_{j \in N}\left(1-\alpha_{j}\right)} .
\end{aligned}
$$

Hence, the expected number of ties at time $X_{(1)}$ among $\left\{X_{i}: i \in N\right\}$ is

$$
\left.E\left[\mid I_{[1]}\right]\right]=\sum_{i \in N} \operatorname{Pr}\left\{X_{i}=X_{(1)}\right\}=\frac{\sum_{i \in N} \alpha_{i}}{1-\prod_{j \in N}\left(1-\alpha_{j}\right)}
$$

We can define the "weighted" random variables $\left\{W_{i}: i \in N\right\}$, where

$$
\forall i \in N, W_{i}=\frac{X_{i}}{E\left[\left|I_{[1]}\right|\right]} \sim \operatorname{Geom}\left(\alpha_{i}, \frac{\omega}{E\left[\left|I_{[1]}\right|\right]}\right) \equiv \operatorname{Geom}\left(\alpha_{i}, \omega \cdot \frac{1-\prod_{j \in N}\left(1-\alpha_{j}\right)}{\sum_{i \in N} \alpha_{i}}\right) .
$$

which are still geometrically distributed random variables with the same success probabilities as their original counterparts $\left\{X_{i}: i \in N\right\}$, but with a reduced time-step. Then,

$$
W_{(1)}=\min \left\{W_{i}: i \in N\right\}=\min \left\{\frac{X_{i}}{E\left[\left|I_{[1]}\right|\right]}: i \in N\right\}=\frac{X_{(1)}}{E\left[\left|I_{[1]}\right|\right]}
$$


takes ties into account by dividing the minimum completion time by the expected number of ties (the corresponding quantity for the continuous case is still simply $Y_{(1)}$, since the probability of ties is zero in this case). The expected value of the weighted minimum for the geometric case coincides with the expected minimum for the exponential case:

$$
E\left[W_{(1)}\right]=E\left[\frac{X_{(1)}}{E\left[\left|I_{[1]}\right|\right]}\right]=\frac{\frac{\omega}{1-\prod_{i \in N}\left(1-\alpha_{i}\right)}}{\frac{\sum_{i \in N} \alpha_{i}}{1-\prod_{j \in N}\left(1-\alpha_{j}\right)}}=\frac{\omega}{\sum_{i \in N} \alpha_{i}}=\left(\sum_{i \in N} \lambda_{i}\right)^{-1}=E\left[Y_{(1)}\right]
$$

We conclude this section by observing that, while the result $E\left[W_{(1)}\right]=E\left[Y_{(1)}\right]$ seems to imply that ties are the cause of Inequality (1) in Section 3, this is not correct, since the inequality holds even when ties are not possible. This can be shown by considering a set of geometric random variables $\left\{X_{i}^{*}: i \in N\right\}$, where

$$
\forall i \in N, X_{i}^{*} \sim \operatorname{Geom}\left(\alpha_{i}^{*}, \omega_{i}\right), E\left[X^{*}\right]=\frac{\omega_{i}}{\alpha_{i}^{*}}=\lambda_{i}^{-1}=E\left[Y_{i}\right]
$$

and, $\forall i \in N, \forall j \in N, i \neq j$, the ratio $\omega_{i} / \omega_{j}$ is not a rational number, hence, it is not possible to find two integers $k_{i}$ and $k_{j}$ that would results in a potential tie at time $k_{i} \omega_{i}=k_{j} \omega_{j}$.

\section{Matching the minimums by time-shifting}

In the previous section, we forced the expectation of the minimums of $\left\{X_{i}: i \in N\right\}$ and $\left\{Y_{i}: i \in N\right\}$ to coincide by reducing the time-step of the geometric distributions, that is, transforming $\left\{X_{i}: i \in N\right\}$ into $\left\{W_{i}: i \in N\right\}$. While the result $E\left[W_{(1)}\right]=E\left[Y_{(1)}\right]$ is appealing, the weighted random variables $\left\{W_{i}: i \in N\right\}$ do not match the original $\left\{Y_{i}: i \in N\right\}$ in expectation. A more interesting result would be to modify our initial set of random variables $\left\{X_{i}: i \in N\right\}$ so that both the individual random variables and the minimum match the corresponding exponential quantities in expectation.

In this section, we accomplish exactly this by introducing the "shifted geometric" distribution, a generalization of both the geometric and modified geometric distribution. Given $0<\alpha \leq 1, \omega>0$, and $\sigma \in \mathbb{R}$, we say that $S$ has a shifted geometric distribution with 
parameters $\alpha, \omega$, and $\sigma, S \sim \operatorname{Shift} \operatorname{Geom}(\alpha, \omega, \sigma)$, if its pmf is

$$
\forall k \in \mathbb{N}, \operatorname{Pr}\{S=k \omega+\sigma\}=\alpha(1-\alpha)^{k}
$$

which implies that its CDF is

$$
\forall t \in \mathbb{R}, \operatorname{Pr}\{S \leq t\}= \begin{cases}1-(1-\alpha)^{\left\lfloor\frac{t-\sigma}{\omega}\right\rfloor+1} & \text { if } t \geq \sigma \\ 0 & \text { otherwise }\end{cases}
$$

and that its expectation is

$$
E[S]=\frac{1-\alpha}{\alpha} \omega+\sigma .
$$

In other words, given a random variable $A \sim \operatorname{ModGeom}(\alpha), \omega>0$, and $\sigma \in \mathbb{R}, S=$ $A \omega+\sigma \sim \operatorname{ShiftGeom}(\alpha, \omega, \sigma)$. Figure 2 shows the relationships between the geometric, modified geometric, shifted geometric, and exponential distributions.

Given $Y \sim \operatorname{Expo}(\lambda)$, we can again consider the condition under which $S$ and $Y$ have the same expectation:

$$
E[S]=\frac{1-\alpha}{\alpha} \omega+\sigma=\lambda^{-1}=E[Y] \Rightarrow \alpha=\frac{\omega \lambda}{1-\sigma \lambda+\omega \lambda} .
$$

Since $\alpha$ is a probability, it can only have values in $[0,1]$. Furthermore, $E[S]=\infty$ when $\alpha=0$, so we exclude this case. Then, $S$ and $Y$ have the same expectation for any choice of $\omega$ and $\sigma$, as long as

$$
0<\alpha=\frac{\omega \lambda}{1-\sigma \lambda+\omega \lambda} \leq 1 \Rightarrow \sigma \leq \lambda^{-1}
$$

and $\alpha$ is set according to Equation (3). A few observations are of particular interest:

- Once the value of $E[S]$ is fixed at $\lambda^{-1}$, decreasing the time-shift $\sigma$ by $\delta$, possibly below zero, causes a decrease in $\alpha$, so that $E[A]$ increases by $\delta / \omega$ and $E[S]=E[A] \omega-\sigma$ remains constant. Since $E[A]$ can be arbitrarily large, this explains why there is no lower bound for $\sigma$.

- If $\sigma=0, S \sim \operatorname{ModGeom}(\alpha, \omega)$.

- If $\omega<\lambda^{-1}$ and $\sigma=\omega, S \sim \operatorname{Geom}(\alpha, \omega)$.

- If $\sigma=\lambda^{-1}, \alpha=1$, hence $S \sim \operatorname{Const}(\sigma) \equiv \operatorname{Const}\left(\lambda^{-1}\right)$. 
Consider now a set of modified geometric random variables with time-step one, $\left\{A_{i}: i \in\right.$ $N\}$ and the set of shifted geometric random variables $\left\{S_{i}: i \in N\right\}$ obtained from them by changing the time-step to $\omega$ and applying a time-shift $\sigma$ :

$$
\forall i \in N, A_{i} \sim \operatorname{ModGeom}\left(\alpha_{i}\right), S_{i}=A_{i} \omega+\sigma \Rightarrow S_{i} \sim \operatorname{Shift} \operatorname{Geom}\left(\alpha_{i}, \omega, \sigma\right)
$$

and set the parameters $\left\{\alpha_{i}: i \in N\right\}$ so that:

$$
\forall i \in N, E\left[S_{i}\right]=\frac{1-\alpha_{i}}{\alpha_{i}} \omega+\sigma=\lambda_{i}^{-1}=E\left[Y_{i}\right] \Rightarrow \alpha_{i}=\frac{\omega \lambda_{i}}{1-\sigma \lambda_{i}+\omega \lambda_{i}} .
$$

Since $\forall i \in N, 0<\alpha_{i} \leq 1$, the maximum value of $\sigma$ is

$$
\sigma \leq \min _{i \in N}\left\{\lambda_{i}^{-1}\right\}=\left(\max _{i \in N}\left\{\lambda_{i}\right\}\right)^{-1}=\lambda_{M A X}^{-1}
$$

The expectation of $S_{(1)}=\min \left\{S_{i}: i \in N\right\}=A_{(1)} \omega+\sigma$ is then

$$
\begin{aligned}
E\left[S_{(1)}\right] & =\frac{\prod_{i \in N}\left(1-\alpha_{i}\right)}{1-\prod_{i \in N}\left(1-\alpha_{i}\right)} \omega+\sigma \\
& =\frac{\prod_{i \in N}\left(1-\frac{\omega \lambda_{i}}{1-\sigma \lambda_{i}+\omega \lambda_{i}}\right)}{1-\prod_{i \in N}\left(1-\frac{\omega \lambda_{i}}{1-\sigma \lambda_{i}+\omega \lambda_{i}}\right)} \omega+\sigma \\
& =\frac{\prod_{i \in N}\left(1-\sigma \lambda_{i}\right)}{\prod_{i \in N}\left(1-\sigma \lambda_{i}+\omega \lambda_{i}\right)-\prod_{i \in N}\left(1-\sigma \lambda_{i}\right)} \omega+\sigma \\
& =\frac{p_{n}}{q_{n}-p_{n}} \omega+\sigma
\end{aligned}
$$

where $p_{n}=\prod_{i \in N}\left(1-\sigma \lambda_{i}\right)$ and $q_{n}=\prod_{i \in N}\left(1-\sigma \lambda_{i}+\omega \lambda_{i}\right)$ satisfy

- $\forall \sigma \leq \lambda_{M_{A X}}^{-1}, p_{n}<q_{n}$.

- $\left.p_{n}\right|_{\sigma=0}=1,\left.q_{n}\right|_{\sigma=0}=\prod_{i \in N}\left(1+\omega \lambda_{i}\right)>1$.

- $\left.p_{n}\right|_{\sigma=\lambda_{M A X}^{-1}}=0$.

- If $\omega<\lambda_{M A X}^{-1},\left.q_{n}\right|_{\sigma=\omega}=1$. 
Theorem 2. There exists a unique value $\sigma^{*} \leq \lambda_{M A X}^{-1}$ for which $E\left[S_{(1)}\right]=E\left[Y_{(1)}\right]$.

Proof. To show the existence of $\sigma^{*}$, it is sufficient to observe that $E\left[S_{(1)}\right]$ is a continuous function of $\sigma$ over $\left(-\infty, \lambda_{M A X}^{-1}\right]$, that

$$
\left.E\left[S_{(1)}\right]\right|_{\sigma=0}=\frac{\omega}{\prod_{i \in N}\left(1+\omega \lambda_{i}\right)-1}<\left(\sum_{i \in N} \lambda_{i}\right)^{-1}=E\left[Y_{(1)}\right]
$$

(this is Inequality (2) in Section 3), and that

$$
\left.E\left[S_{(1)}\right]\right|_{\sigma=\lambda_{M A X}^{-1}}=\lambda_{M A X}^{-1}>\left(\sum_{i \in N} \lambda_{i}\right)^{-1}=E\left[Y_{(1)}\right] .
$$

Hence, by continuity, there must exist a value $\sigma^{*} \in\left(0, \lambda_{M A X}^{-1}\right)$ satisfying

$$
\left.E\left[S_{(1)}\right]\right|_{\sigma=\sigma^{*}}=E\left[Y_{(1)}\right]
$$

Furthermore, if $\omega<\lambda_{M A X}^{-1}$,

$$
\left.E\left[S_{(1)}\right]\right|_{\sigma=\omega}=\frac{\prod_{i \in N}\left(1-\omega \lambda_{i}\right)}{1-\prod_{i \in N}\left(1-\omega \lambda_{i}\right)} \omega+\omega=\frac{\omega}{1-\prod_{i \in N}\left(1-\omega \lambda_{i}\right)}>\left(\sum_{i \in N} \lambda_{i}\right)^{-1}=E\left[Y_{(1)}\right]
$$

(this is Inequality (1) in Section 3), hence, in general, $\sigma^{*} \in\left(0, \min \left\{\omega, \lambda_{M A X}^{-1}\right\}\right)$.

We prove the uniqueness of $\sigma^{*}$ by induction on $n$, showing that $E\left[S_{(1)}\right]$ is a strictly increasing function of $\sigma$ over $\left(-\infty, \lambda_{M A X}^{-1}\right]$, hence we make the index $n$ explicit in $E\left[S_{(1)}\right]$ by writing

$$
E\left[S_{(1, n)}\right]=\min \left\{S_{i}: i \in N\right\}
$$

Base step: For $n=2$,

$$
\begin{aligned}
E\left[S_{(1,2)}\right] & =\frac{\left(1-\sigma \lambda_{1}\right)\left(1-\sigma \lambda_{2}\right)}{\left(1-\sigma \lambda_{1}+\omega \lambda_{1}\right)\left(1-\sigma \lambda_{2}+\omega \lambda_{2}\right)-\left(1-\sigma \lambda_{1}\right)\left(1-\sigma \lambda_{2}\right)} \omega+\sigma \\
& =\frac{1+\sigma \lambda_{1} \lambda_{2}(\omega-\sigma)}{\lambda_{1}+\lambda_{2}+\lambda_{1} \lambda_{2}(\omega-2 \sigma)}
\end{aligned}
$$

and

$$
\begin{aligned}
\frac{d E\left[S_{(1,2)}\right]}{d \sigma} & =\frac{\lambda_{1} \lambda_{2}\left(\omega\left(\lambda_{1}+\lambda_{2}-2 \lambda_{1} \lambda_{2} \sigma\right)+2\left(1-\sigma \lambda_{1}-\sigma \lambda_{2}+\sigma^{2} \lambda_{1} \lambda_{2}\right)+\omega^{2} \lambda_{1} \lambda_{2}\right)}{\left(\lambda_{1}+\lambda_{2}+\lambda_{1} \lambda_{2}(\omega-2 \sigma)\right)^{2}} \\
& =\frac{\lambda_{1} \lambda_{2}(\overbrace{\omega\left(\lambda_{1}\left(1-\sigma \lambda_{2}\right)+\lambda_{2}\left(1-\sigma \lambda_{1}\right)\right)+2\left(1-\sigma \lambda_{1}\right)\left(1-\sigma \lambda_{2}\right)}^{-1}+\omega^{2} \lambda_{1} \lambda_{2})}{\left(\lambda_{1}+\lambda_{2}+\lambda_{1} \lambda_{2}(\omega-2 \sigma)\right)^{2}}>0 .
\end{aligned}
$$


In particular,

$$
\lim _{\sigma \rightarrow-\infty} E\left[S_{(1,2)}\right]=-\infty \quad \text { and } \quad \lim _{\sigma \rightarrow-\infty} \frac{d E\left[S_{(1,2)}\right]}{d \sigma}=\frac{1}{2}
$$

and

$$
\left.E\left[S_{(1,2)}\right]\right|_{\sigma=\lambda_{M A X}^{-1}}=\lambda_{M A X}^{-1} \quad \text { and }\left.\quad \frac{d E\left[S_{(1,2)}\right]}{d \sigma}\right|_{\sigma=\lambda_{M A X}^{-1}}=\frac{\omega}{\omega+\lambda_{M I N}^{-1}-\lambda_{M A X}^{-1}} \in\left(\frac{\omega}{\omega+\lambda_{M I N}^{-1}}, 1\right]
$$

where $\lambda_{M I N}=\min \left\{\lambda_{1}, \lambda_{2}\right\}$.

Inductive Hypothesis: Assume that, for a given $n$,

$$
E\left[S_{(1, n)}\right]=\frac{p_{n}}{q_{n}-p_{n}} \omega+\sigma
$$

is a strictly increasing function of $\sigma$ over $\left(-\infty, \lambda_{M A X}^{-1}\right]$, that is

$$
\frac{d E\left[S_{(1, n)}\right]}{d \sigma}=\frac{p_{n}^{\prime}\left(q_{n}-p_{n}\right)-p_{n}\left(q_{n}^{\prime}-p_{n}^{\prime}\right)}{\left(q_{n}-p_{n}\right)^{2}} \omega+1=\frac{p_{n}^{\prime} q_{n}-p_{n} q_{n}^{\prime}}{\left(q_{n}-p_{n}\right)^{2}} \omega+1>0
$$

which implies

$$
\forall \sigma \in\left(-\infty, \lambda_{M A X}^{-1}\right], \omega\left(p_{n}^{\prime} q_{n}-p_{n} q_{n}^{\prime}\right)>-\left(q_{n}-p_{n}\right)^{2}
$$

Inductive Step: Then the same holds for $n+1$, that is,

$$
E\left[S_{(1, n+1)}\right]=\frac{p_{n+1}}{q_{n+1}-p_{n+1}} \omega+\sigma=\frac{p_{n}\left(1-\sigma \lambda_{n+1}\right)}{q_{n}\left(1-\sigma \lambda_{n+1}+\omega \lambda_{n+1}\right)-p_{n}\left(1-\sigma \lambda_{n+1}\right)} \omega+\sigma,
$$

where $\lambda_{n+1}$ is the rate of the $(n+1)$-th exponential random variable, is a strictly increasing function of $\sigma$ over $\left(-\infty, \max \left\{\lambda_{M_{A X}}, \lambda_{n+1}\right\}^{-1}\right]$, that is

$$
\begin{aligned}
\frac{d E\left[S_{(1, n+1)}\right]}{d \sigma} & =\frac{\left(1-\sigma \lambda_{n+1}\right)\left(1-\sigma \lambda_{n+1}+\omega \lambda_{n+1}\right) \overbrace{\left(p_{n}^{\prime} q_{n}-p_{n} q_{n}^{\prime}\right) \omega}^{>-\left(q_{n}-p_{n}\right)^{2} \text { for } \sigma \leq \lambda_{M A X}^{-1}}-\lambda_{n+1}^{2} \omega^{2} q_{n} p_{n}}{\left(q_{n}\left(1-\sigma \lambda_{n+1}+\omega \lambda_{n+1}\right)-p_{n}\left(1-\sigma \lambda_{n+1}\right)\right)^{2}}+1 \\
& >\frac{\left(1-\sigma \lambda_{n+1}\right)\left(1-\sigma \lambda_{n+1}+\omega \lambda_{n+1}\right)\left(-\left(q_{n}-p_{n}\right)^{2}\right)-\lambda_{n+1}^{2} \omega^{2} q_{n} p_{n}}{\left(q_{n}\left(1-\sigma \lambda_{n+1}+\omega \lambda_{n+1}\right)-p_{n}\left(1-\sigma \lambda_{n+1}\right)\right)^{2}}+1 \\
& =\frac{\lambda_{n+1} \omega\left(q_{n}-p_{n}\right)\left(\left(q_{n}+p_{n}\right)\left(1-\sigma \lambda_{n+1}\right)+\lambda_{n+1} \omega q_{n}\right)}{\left(q_{n}\left(1-\sigma \lambda_{n+1}+\omega \lambda_{n+1}\right)-p_{n}\left(1-\sigma \lambda_{n+1}\right)\right)^{2}}>0
\end{aligned}
$$

since, $\sigma<\lambda_{n+1}^{-1}$ and $\forall \sigma \in\left(-\infty, \max \left\{\lambda_{M_{A X}}, \lambda_{n+1}\right\}^{-1}\right], q_{n}>p_{n}$. QED. 
We might now ask whether this value $\sigma^{*}$ for which $E\left[S_{(1)}\right]=E\left[Y_{(1)}\right]$ is such that the other order statistics coincide as well, that is, whether

$$
\forall i \in N, i>1,\left.E\left[S_{(i)}\right]\right|_{\sigma=\sigma^{*}}=E\left[Y_{(i)}\right]
$$

This is indeed true for $n=2$, since

$$
E\left[S_{(1)}\right]+E\left[S_{(2)}\right]=E\left[S_{1}\right]+E\left[S_{2}\right]=E\left[Y_{1}\right]+E\left[Y_{2}\right]=E\left[Y_{(1)}\right]+E\left[Y_{(2)}\right]
$$

implies that, whenever $E\left[S_{(1)}\right]=E\left[Y_{(1)}\right]$,

$$
E\left[S_{(2)}\right]=E\left[Y_{(2)}\right]
$$

Unfortunately, this is not true in general for $n \geq 3$, as it can be seen considering the homogeneous case. When $\forall i \in N, \lambda_{i}=\lambda$,

$$
\begin{aligned}
\operatorname{Pr}\left\{S_{(2)}>k \omega+\sigma\right\} & =\operatorname{Pr}\left\{A_{(2)}>k\right\} \\
& =\operatorname{Pr}\left\{\left(\forall i \in N, A_{i}>k\right) \vee\left(\bigvee_{i \in N}\left(A_{i} \leq k \wedge \forall j \in N, j \neq i, A_{j}>k\right)\right)\right\} \\
& =\operatorname{Pr}\left\{\forall i \in N, A_{i}>k\right\}+\sum_{i \in N} \operatorname{Pr}\left\{A_{i} \leq k \wedge \forall j \in N, j \neq i, A_{j}>k\right\} \\
& =\left((1-\alpha)^{n}\right)^{k+1}+n\left(1-(1-\alpha)^{k+1}\right)\left((1-\alpha)^{n-1}\right)^{k+1} \\
& =n(1-\alpha)^{(n-1)(k+1)}-(n-1)(1-\alpha)^{n(k+1)}
\end{aligned}
$$

and

$$
\begin{aligned}
E\left[A_{(2)}\right] & =\sum_{k=0}^{\infty} \operatorname{Pr}\left\{A_{(2)}>k\right\} \\
& =\sum_{k=0}^{\infty} n(1-\alpha)^{(n-1)(k+1)}-(n-1)(1-\alpha)^{n(k+1)} \\
& =\frac{n(1-\alpha)^{n-1}}{1-(1-\alpha)^{n-1}}-\frac{(n-1)(1-\alpha)^{n}}{1-(1-\alpha)^{n}}
\end{aligned}
$$

Hence, considering $S^{(2)}=A_{(2)} \omega+\sigma$ and substituting $\alpha$ from (3),

$$
E\left[S_{(2)}\right]=\left(\frac{n(1-\sigma \lambda)^{n-1}}{(1-\sigma \lambda-\omega \lambda)^{n-1}-(1-\sigma \lambda)^{n-1}}-\frac{(n-1)(1-\sigma \lambda)^{n}}{(1-\sigma \lambda-\omega \lambda)^{n}-(1-\sigma \lambda)^{n}}\right) \omega+\sigma,
$$

while, due to the absence of memory of the exponential distribution,

$$
E\left[Y_{(2)}\right]=(n \lambda)^{-1}+((n-1) \lambda)^{-1}
$$

It can be easily verified numerically, for example when $n=3, \omega=1 / 2, \lambda=1$, that the only real root of $E\left[S_{(1)}\right]=E\left[Y_{(1)}\right]$ is $\sigma \approx 0.173927$, while the only real root less than $\lambda^{-1}$ of $E\left[S_{(2)}\right]=E\left[Y_{(2)}\right]$ is $\sigma \approx 0.346961$. 


\section{Conclusion}

We have shown how, if the random variables $\left\{X_{i}: i \in N\right\},\left\{Y_{i}: i \in N\right\}$, and $\left\{Z_{i}: i \in N\right\}$ model the same set of $n$ concurrent activities using geometric, exponential, or modified geometric distributions, respectively, with given expectations $\left\{\lambda_{i}^{-1}: i \in N\right\}$, the minimum in each set has a different expected value: $E\left[X_{(1)}\right]>E\left[Y_{(1)}\right]>E\left[Z_{(1)}\right]$. Stochastic variability is employed to justify the result.

We then consider two different ways to match the expectation of the minimums. First, by taking into account the possibility of ties in the geometric case, we define the "weighted minimum" $W_{(1)}$, and obtain $E\left[W_{(1)}\right]=E\left[Y_{(1)}\right]$, but this operation corresponds to decreasing the time-step of the individual geometric distributions, hence their expectation. Alternatively,

we introduce the "shifted geometric distribution", which a generalizes both the geometric and the modified geometric. We can then define a set of shifted geometric random variables $\left\{S_{i}: i \in N\right\}$, which match in expectation the exponential random variables both individually, $E\left[S_{i}\right]=E\left[Y_{i}\right]=\lambda_{i}^{-1}$, and their minimum, $E\left[S_{(1)}\right]=E\left[Y_{(1)}\right]$.

\section{Refences}

Margolin, B.H. and H.S. Winokur, Jr. (1967), Exact moments of the order statistics of the geometric distribution and their relation to inverse sampling and reliability of redundant systems, Journal of the American Statistical Association, 62, 915-925.

Ross, S.M. (1983), Stochastic Processes (Wiley, New York, NY).

Trivedi, K.S. (1982), Probability 8 Statistics with Reliability, Queueing, and Computer Science Applications (Prentice-Hall, Englewood Cliffs, N.J).

Uppuluri, V.R.R. (1964), A characterization of the geometric distribution, Annals of Mathematical Statistics, (Abstract 61) 35, 1841. 

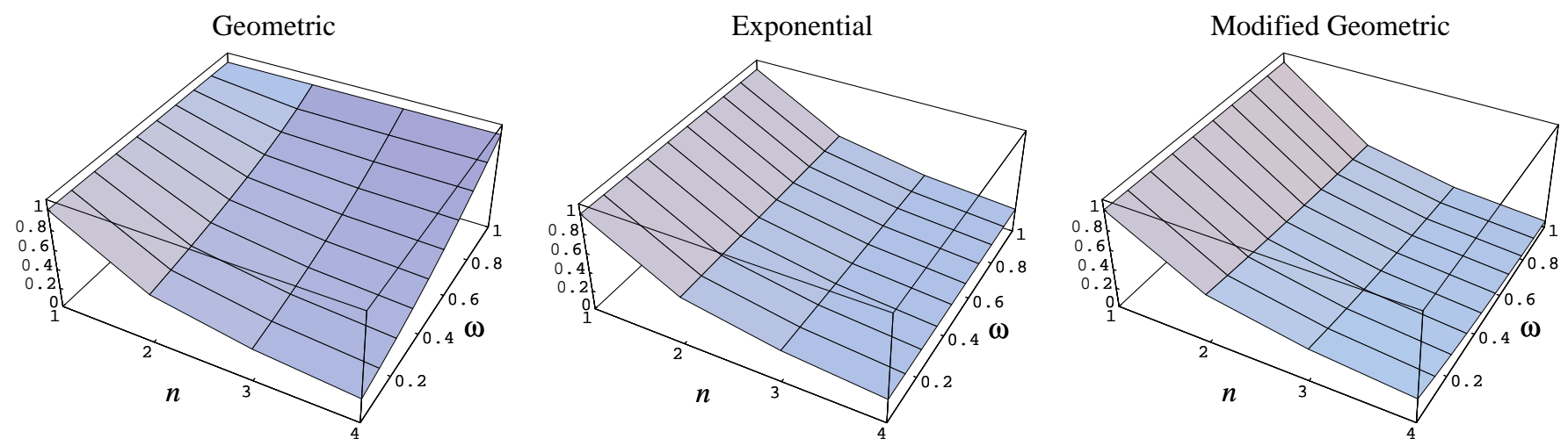

Figure 1: $E\left[X_{(1)}\right], E\left[Y_{(1)}\right]$, and $E\left[Z_{(1)}\right]\left(n=1,2,3,4, \forall i, \lambda_{i}=1\right)$ as a function of $\omega$.

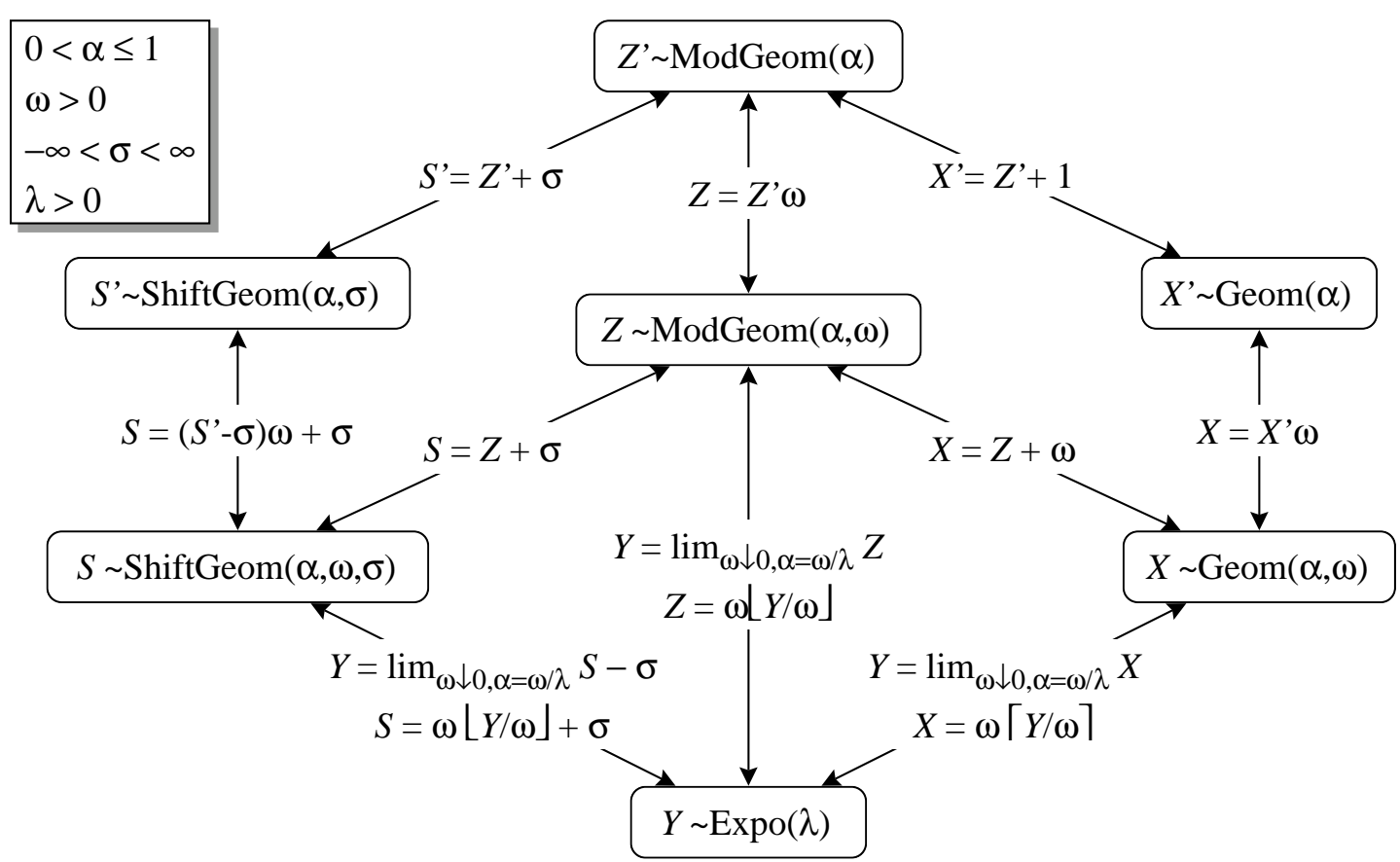

Figure 2: Relationships between the distributions discussed in this paper. 